\title{
Plasma chemokines as immune biomarkers for diagnosis of pediatric tuberculosis
}

\author{
Nathella Pavan Kumar ${ }^{2 *}$, Syed Hissar ${ }^{2 *}$, Kannan Thiruvengadam², Velayuthum V. Banurekha ${ }^{2}$, Sarath Balaji ${ }^{3}$, \\ S. Elilarasi ${ }^{3}$, N. S. Gomathi², J. Ganesh ${ }^{4}$, M. A. Aravind ${ }^{4}$, Dhanaraj Baskaran², Srikanth Tripathy², \\ Soumya Swaminathan ${ }^{2,5}$ and Subash Babu ${ }^{1,6}$
}

\begin{abstract}
Background: Diagnosing tuberculosis (TB) in children is challenging due to paucibacillary disease, and lack of ability for microbiologic confirmation. Hence, we measured the plasma chemokines as biomarkers for diagnosis of pediatric tuberculosis.

Methods: We conducted a prospective case control study using children with confirmed, unconfirmed and unlikely TB. Multiplex assay was performed to examine the plasma CC and CXC levels of chemokines.

Results: Baseline levels of CCL1, CCL3, CXCL1, CXCL2 and CXCL10 were significantly higher in active TB (confirmed TB and unconfirmed TB) in comparison to unlikely TB children. Receiver operating characteristics curve analysis revealed that CCL1, CXCL1 and CXCL10 could act as biomarkers distinguishing confirmed or unconfirmed TB from unlikely TB with the sensitivity and specificity of more than $80 \%$. In addition, combiROC exhibited more than $90 \%$ sensitivity and specificity in distinguishing confirmed and unconfirmed TB from unlikely TB. Finally, classification and regression tree models also offered more than $90 \%$ sensitivity and specificity for CCL1 with a cutoff value of $28 \mathrm{pg} / \mathrm{ml}$, which clearly classify active TB from unlikely TB. The levels of CCL1, CXCL1, CXCL2 and CXCL10 exhibited a significant reduction following anti-TB treatment.
\end{abstract}

Conclusion: Thus, a baseline chemokine signature of CCL1/CXCL1/CXCL10 could serve as an accurate biomarker for the diagnosis of pediatric tuberculosis.

Keywords: Pediatric tuberculosis, Chemokines, Biomarkers

\section{Background}

Diagnosis of active tuberculosis (TB) in children provides a key challenge, because most of the clinical symptoms of TB in children are non-specific with signs and symptoms that resemble common pediatric illnesses, including pneumonia and malnutrition and therefore clinical diagnosis is unreliable [1]. Using respiratory specimens for the diagnosis of bacterial confirmation in young children is difficult because they are unable to expectorate

*Correspondence: pavankumarn@nirt.res.in; drsyed@rediffmail.com ${ }^{2}$ ICMR-National Institute for Research in Tuberculosis, Chennai, India Full list of author information is available at the end of the article sputum. In addition, the diagnostic yields in the sputum sample are not high due to the paucibacillary nature of the disease $[2,3]$. Thus, a novel, non-sputum-based point-of-care (POC) diagnostic tool that could give an early and precise diagnosis of TB disease in children is urgently required. A recent WHO report states the need for a rapid biomarker based non-sputum-dependent diagnostic test for the diagnosis of pulmonary TB [4]. Children are clinically crucial populations with greater susceptibility to tuberculosis, and mechanisms of protection are still unclear [5]. WHO reports that among the 10 million new cases of active TB each year, approximately $10 \%$ happen in children less than 15 years of age 
[6]. Mycobacterium tuberculosis (M.tb) exposed children acquire infection, which is described as immunological evidence of sensitisation to M.tb, and some of these children may develop TB disease with signs and symptoms with microbiological evidence of disease [7]

It has also been reported in a few studies that IP-10 detection could be useful for diagnosing active TB and LTBI in children [8] and even in the adult population, IP-10 and MCP-2 [9] can be used as biomarkers for tuberculosis. In addition, some studies with smaller sample size have shown that IP-10, IFN- $\gamma$ and IL-2 can be used as a diagnostic tool for pediatric tuberculosis [10]. However, most of these studies used only the antigen specific chemokine or cytokine response for diagnosis.

Better-quality methods for diagnosing pediatric TB are thus immediately needed and immunological biomarker assays can bring light to this problem. Chemokines are chemoattractant molecules that control immunological responses and have been widely considered for their utility as diagnostic biomarkers of adult TB [11]. Results of our previous published studies in adults showed that plasma chemokines are significantly elevated in an active TB group and can significantly discriminate active TB from latent $\mathrm{TB}$ or healthy controls [12]. In this proofof-concept study, we aimed to determine the plasma concentrations of CC (CCL1, CCL2, CCL3, CCL4 and CCL11) and CXC (CXCL1 CXCL2, CXCL9, CXCL10 and CXCL11) chemokines as markers capable of discriminating among children who were microbiology positive (confirmed TB) or those negative but clinically diagnosed and treated (unconfirmed TB) compared with unlikely $\mathrm{TB}$ children in a prospectively recruited cohort for the study.

\section{Methods}

\section{Study population and procedures}

We conducted a prospective case control study using children with confirmed, unconfirmed and unlikely TB. All admitted and out patients in the pediatric wards of Institute of Child Health and Hospital for Children, Chennai and Government Stanley Medical College and Hospital, Chennai, were screened. Children aged less than 15 years of age with symptoms suggestive of Intrathoracic tuberculosis were enrolled between February 2016 and March 2018. Of the 195 children screened, 167 children were recruited which includes 44 children who were microbiology positive (confirmed TB) for M.tb, 47 children who were microbiology negative (unconfirmed TB), 76 children with other respiratory ailments with tuberculin skin Test (TST) positive or negative as unlikely TB controls. Two sputum samples were collected from each child on two consecutive days and gastric aspirates were collected for children aged $<5$. All children in the study population underwent sputum smear and culture (or Xpert MTB/RIF) and Tuberculin Skin Test (TST).

Definition of clinical groups [13]

Confirmed TB: Microbiologically positive for M.tb. Positive for Xpert MTB/RIF or smear or culture for M.tb.

Unconfirmed TB: Clinical features suggestive of TB or abnormal chest X-ray or history of household TB contact or response to anti-tuberculosis treatment (ATT) [at least two of the criteria were met].

Unlikely TB: Children who had a differential diagnosis apart from TB and who were either negative or positive for TST results were considered as controls.

Unlikely TB children were children who were suspected of having TB but clinically diagnosed as having other respiratory illness like COPD, viral pneumonia, bacterial pneumonia or Asthma/wheeze, empirically treated with antibiotics/antivirals/anti asthmatic medication and followed up for a period of 8 weeks for resolutions of symptoms, resolution of chest $\mathrm{x}$-ray abnormalities and confirmed as TB culture negative.

At the time of enrolment, all active TB cases (Confirmed and Unconfirmed TB together) had no record of prior TB disease or ATT. A positive TST result was defined as an induration at the site of tuberculin inoculation of at least $10 \mathrm{~mm}$ in diameter. Plasma samples were collected from all of the above children in a consecutive sampling method. From the confirmed TB and unconfirmed TB children blood samples were collected before starting the anti-tuberculosis treatment. All the blood samples were collected in sodium heparin tubes. Collected blood samples were transported within $2 \mathrm{~h}$ to the Immunology lab for processing and further plasma samples were stored in $-80^{\circ} \mathrm{C}$ freezer. All children were HIV negative. All confirmed and unconfirmed TB children were administered ATT for 6 months. At 6 months following ATT initiation, fresh plasma samples were obtained from a subset of confirmed TB $(n=24)$ and unconfirmed TB $(n=23)$ children. All children with active TB were culture negative and symptom free at the end of ATT. The demographic and epidemiological data have been previously reported [14].

\section{Multiplex chemokine assay}

Plasma levels of CC (CCL1, CCL2, CCL3, CCL4 and CCL11) and CXC (CXCL1 CXCL2, CXCL9, CXCL10 and CXCL11) chemokines were measured using the Luminex Magpix multiplex cytokine assay system (BioRad, Hercules, CA, USA). Luminex Human Chemokine Magnetic Assay kit ( $\mathrm{R} \& \mathrm{D}$ systems, USA) was used to measure the chemokine levels. All the assays were performed in the stored samples. The lowest detection limits were as follows: CCL1, $4.5 \mathrm{pg} / \mathrm{mL}$; CCL2, $31.8 \mathrm{pg} /$ 
mL; CCL3, 30.9 pg/mL; CCL4, $133.4 \mathrm{pg} / \mathrm{mL}$; CCL11, $21.6 \mathrm{pg} / \mathrm{mL}$; CXCL1, $49.2 \mathrm{pg} / \mathrm{mL}$; CXCL2, $49.2 \mathrm{pg} / \mathrm{mL}$; CXCL9, $600 \mathrm{pg} / \mathrm{mL}$, CXCL10, $2.8 \mathrm{pg} / \mathrm{mL}$ and CXCL11, $21.6 \mathrm{pg} / \mathrm{mL} . \mathrm{N}=44$ samples were analysed per batch in duplicates, and lab personal were blinded to the clinical groups. All the assays were performed according to the manufacturer's instructions.

\section{Statistical analysis}

Geometric means (GM) were used for measurements of central tendency. Statistically significant differences between confirmed TB, unconfirmed TB and unlikely TB children were analysed using the Kruskal-Wallis test with Dunn's multiple comparisons. Wilcoxon signed rank test was used to compare cytokine concentrations before and after ATT. Receiver Operator Characteristics (ROC) curves were used to determine accuracy of each candidate chemokine immune biomarker to distinguish confirmed TB, unconfirmed TB and unlikely TB. Analyses were performed using Graph-Pad PRISM Version 9.0 (GraphPad Software, CA, USA). Values below the detection limits were handled using the least squared (ordinary) fit method to imputed the quantitative value and as such included in graphs and for the statistical analysis [15]. Computation and selection of optimal biomarker combinations by integrative ROC were analysed using a freely available web application (http://CombiROC.eu) CombiROC v.1.2. [16]. Classification and regression trees (CART) model were employed to identify the cut-off values for the biomarkers which separate the active TB children from those with unlikely TB. The analysis was done using the R (R Foundation for Statistical Computing, Vienna, Austria) software.

\section{Results}

\section{Participant categorisation}

The recruitment algorithm for the children is shown in Fig. 1. Of the 195 children screened, 167 were recruited. The demographics of the children are shown in Table 1. We had 20 children below 5 years, 8 children between 5 and 10 and 16 children between 10 and 12 years in the confirmed TB group; and 21 children below 5 years, 6 children between 5 and 10 years and 20 children between 10 and 12 years in the unconfirmed TB group. All children in the confirmed and unconfirmed TB group were TST positive.

\section{Plasma levels of chemokines are elevated in children with active TB disease}

To determine the levels of plasma chemokines in children with active TB disease and with no TB disease (but with other respiratory illness), we measured the plasma

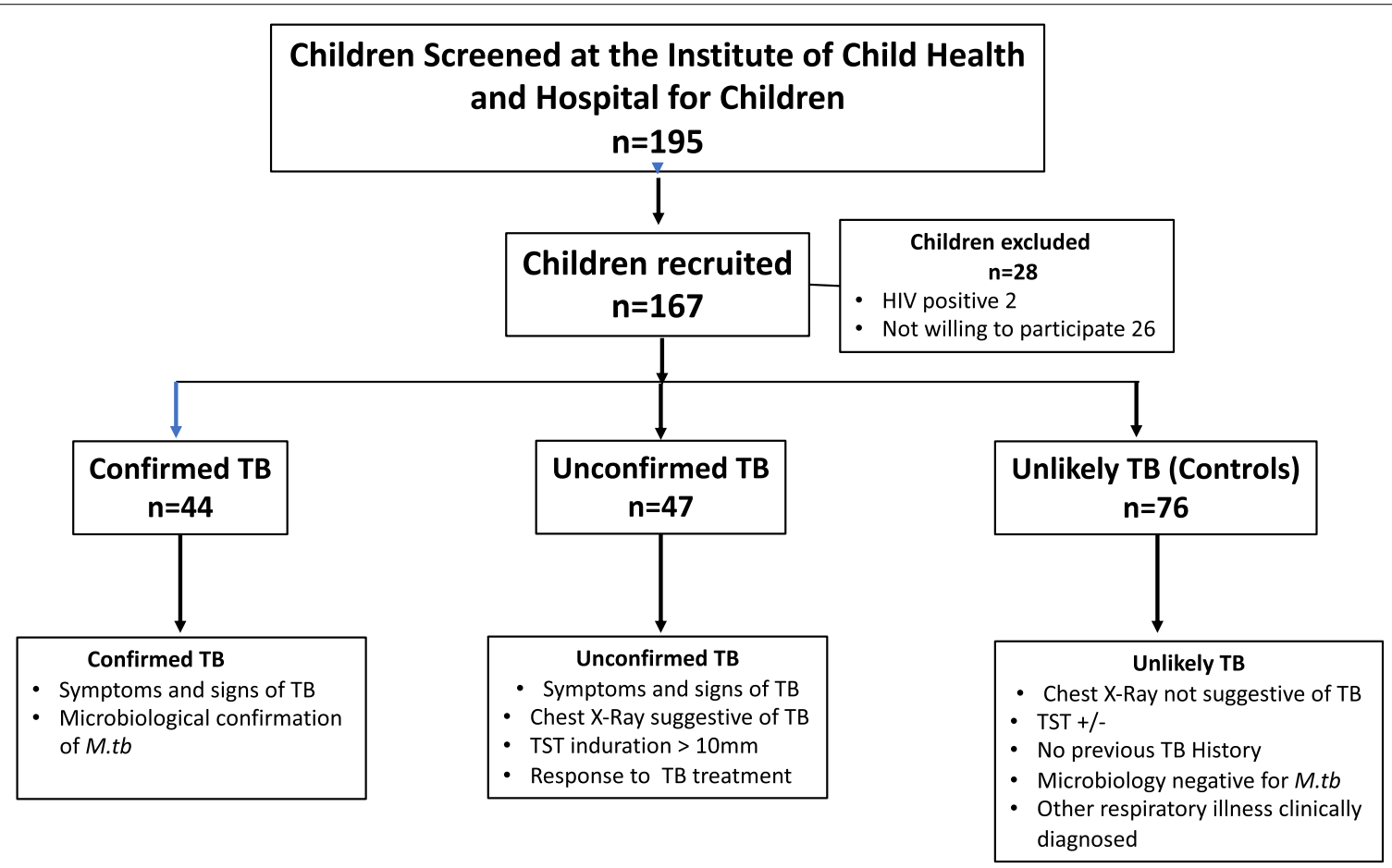

Fig. 1 Outline of participant categorisation. In the study cohort ( $N=167)$, plasma samples were collected from children who were microbiology positive (confirmed TB) or those negative (unconfirmed TB) for M.tb but with symptoms that suggested tuberculosis at baseline and at the end of ATT. Children with other respiratory ailments as unlikely TB were also studied at baseline as controls 
Table 1 Study demographics

\begin{tabular}{llll}
\hline Demographic characteristic & Confirmed TB & Unconfirmed TB & Unlikely TB \\
\hline Number of subjects recruited & 44 & 47 & 76 \\
Gender (male / female) & $16 / 28$ & $28 / 19$ & $36 / 40$ \\
Median age (range) (in years) & $7(1-13)$ & $8(1-12)$ & $6(1-14)$ \\
Bacterial burden: & $11 / 33 / 0$ & $0 / 0 / 47$ & - \\
High burden/ low burden / no burden & & & $45 / 31$ \\
Tuberculin skin test: (positive/negative) & $44 / 0$ & $35 / 12$ & \\
\hline
\end{tabular}

levels of CC (CCL1, CCL2, CCL3, CCL4 and CCL11) and CXC (CXCL1 CXCL2, CXCL9, CXCL10 and CXCL11) in children with confirmed TB $(\mathrm{n}=44)$, unconfirmed TB $(\mathrm{n}=47)$ and unlikely $\mathrm{TB}(\mathrm{n}=76)$ (Fig. 2). As shown in Fig. 2, the plasma levels of $\mathrm{CC}$ and $\mathrm{CXC}$ chemokinesCCL1 (Geometric Mean (GM) of $57.2 \mathrm{pg} / \mathrm{ml}$ in confirmed TB vs $39.14 \mathrm{pg} / \mathrm{ml}$ in unconfirmed $\mathrm{TB}$ and $14.01 \mathrm{pg} / \mathrm{ml}$ in Unlikely TB), CCL3 (GM of $81.7 \mathrm{pg} / \mathrm{ml}$ in confirmed $\mathrm{TB}$ vs $73.4 \mathrm{pg} / \mathrm{ml}$ in unconfirmed $\mathrm{TB}$ and $51 \mathrm{pg} / \mathrm{ml}$ in Unlikely TB), CXCL1 (GM of $157.6 \mathrm{pg} / \mathrm{ml}$ in confirmed $\mathrm{TB}$ vs $48.2 \mathrm{pg} / \mathrm{ml}$ in unconfirmed $\mathrm{TB}$ and $21.5 \mathrm{pg} / \mathrm{ml}$ in Unlikely TB), CXCL2 (GM of $809.1 \mathrm{pg} / \mathrm{ml}$ in confirmed $\mathrm{TB}$ vs $514.4 \mathrm{pg} / \mathrm{ml}$ in unconfirmed TB and $147.6 \mathrm{pg} /$ $\mathrm{ml}$ in Unlikely TB) and CXCL10 (GM of $102.7 \mathrm{pg} / \mathrm{ml}$ in confirmed TB vs $62.8 \mathrm{pg} / \mathrm{ml}$ in unconfirmed TB and $9.8 \mathrm{pg} / \mathrm{ml}$ in Unlikely $\mathrm{TB}$ ) were significantly higher in children with confirmed and unconfirmed TB compared to unlikely TB children.

\section{Plasma chemokines can robustly distinguish confirmed and unconfirmed TB from unlikely TB}

To determine the discriminatory power of plasma $\mathrm{CC}$ and $\mathrm{CXC}$ chemokines in distinguishing children with confirmed and unconfirmed TB from unlikely TB, we performed ROC analysis of CCL1, CXCL1 and CXCL10 in confirmed and unconfirmed TB vs. unlikely TB children and confirmed vs unconfirmed TB (Fig. 3). As shown in Fig. 3A, CCL1 (AUC 0.9913, sensitivity 97\%,

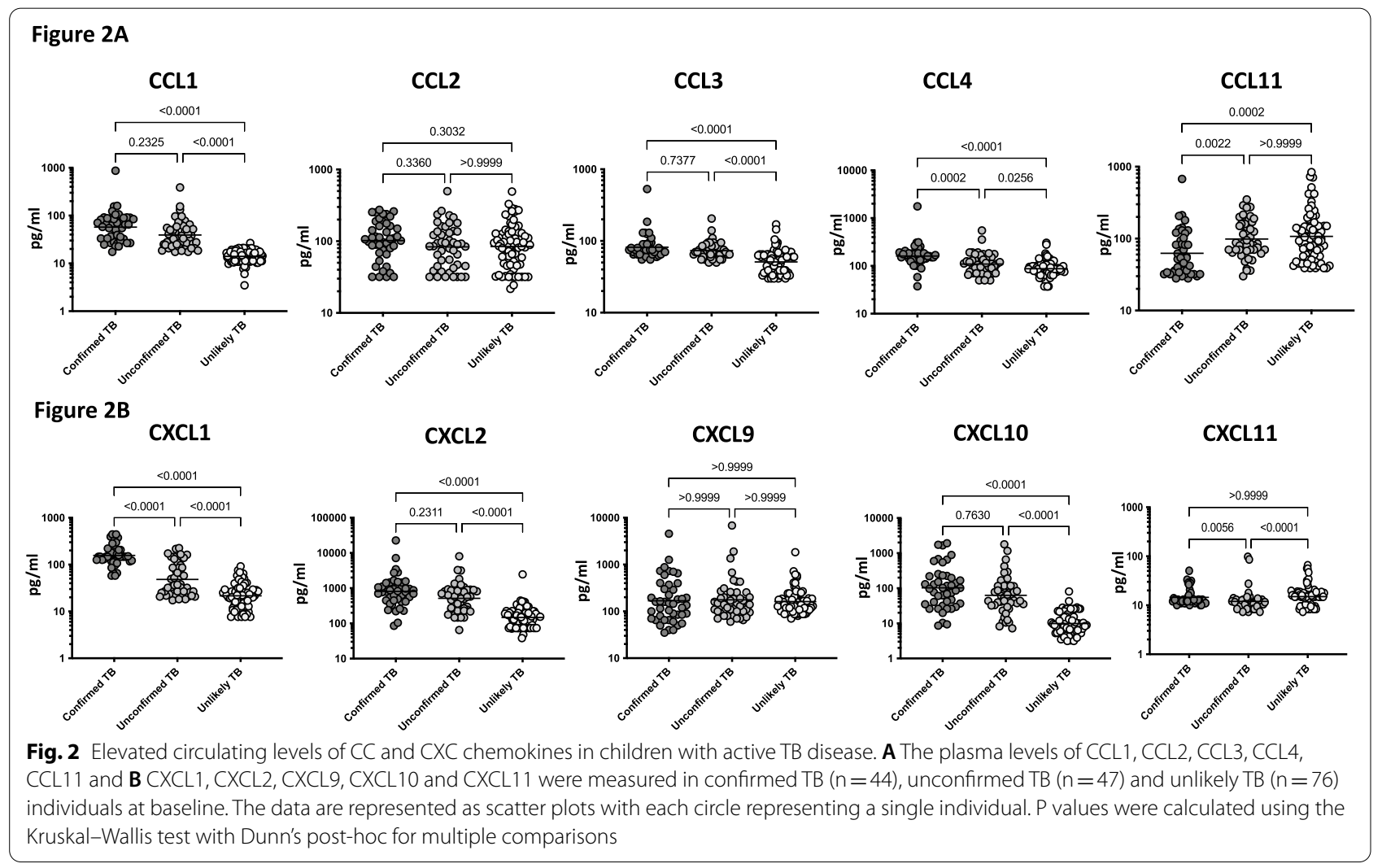




\section{Figure 3A : Confirmed TB vs Unlikely TB}

CCL1

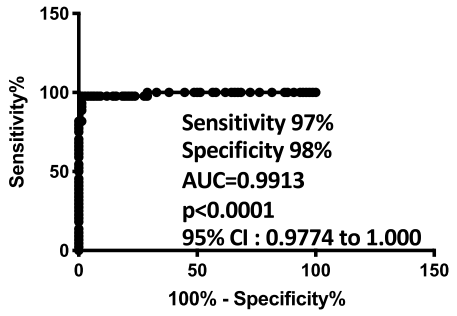

Figure 3B : Unconfirmed TB vs Unlikely TB

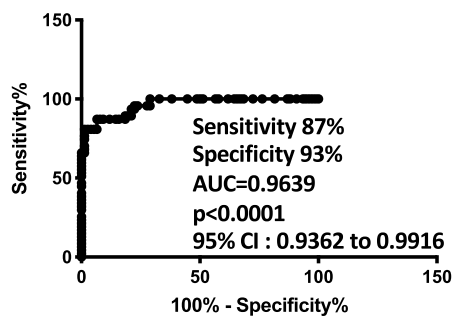

Figure 3C : Confirmed TB vs Unconfirmed TB

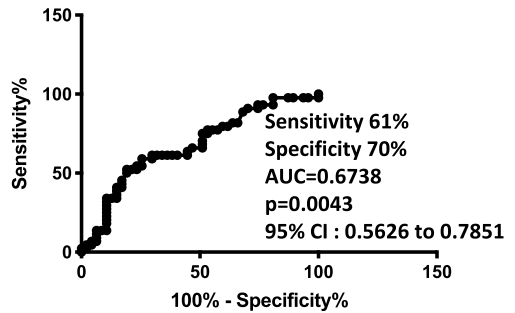

CXCL1
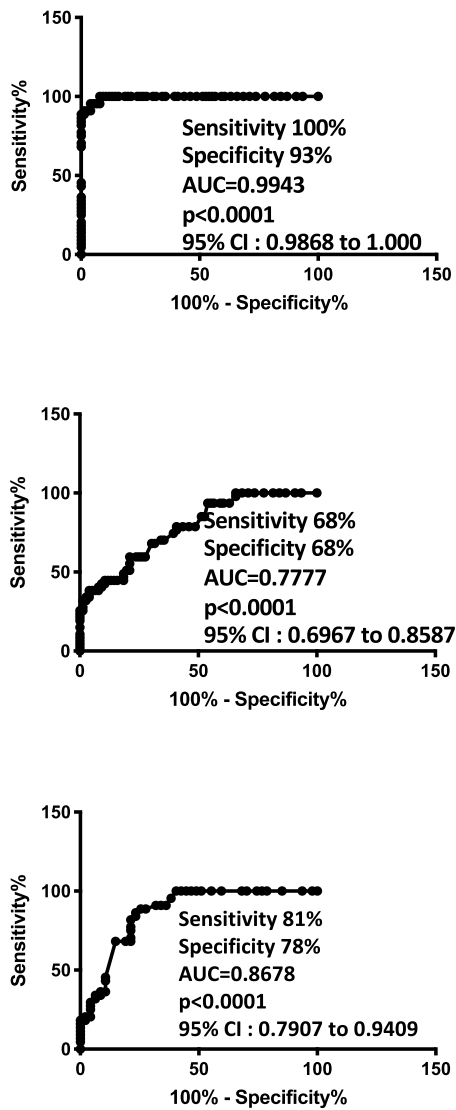

CXCL10

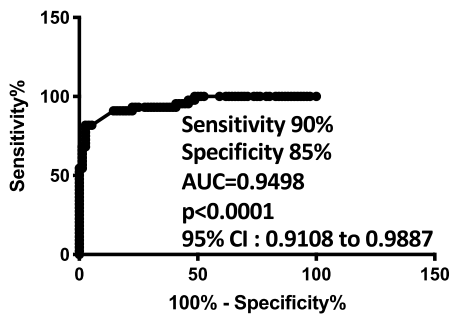

Fig. 3 ROC analysis to estimate the discriminatory power of CC and CXC chemokines in children with active TB disease and unlikely TB. ROC analysis to estimate the sensitivity, specificity and AUC was performed using CCL1, CXCL1 and CXCL10 to estimate the capacity of these factors to distinguish individuals with (A) confirmed TB vs. unlikely TB (B) Unconfirmed TB vs. unlikely TB (C) confirmed TB vs. unconfirmed TB. ROC receiver operator characteristics

specificity 98\%), CXCL1 (AUC 0.9943, sensitivity 100\%, specificity 93\%), CXCL10 (AUC 0.9498, sensitivity 90\%, specificity $85 \%$ ) exhibited significant discriminatory power with high AUC values, sensitivity and specificity in discriminating confirmed TB from unlikely TB children. Similarly, as shown in Fig. 3B, CCL1 (AUC 0.9639, sensitivity $87 \%$, specificity 93\%), CXCL1 (AUC 0.777, sensitivity 68\%, specificity 68\%), CXCL10 (AUC 0.9187, sensitivity $85 \%$, specificity $71 \%$ ), exhibited significant discriminatory power with high AUC values, sensitivity and specificity in discriminating unconfirmed TB from unlikely TB children. Finally, as shown in Fig. 3C, CCL1 (AUC 0.6738, sensitivity 61\%, specificity 70\%), CXCL1 (AUC 0.8678 , sensitivity $81 \%$, specificity 78\%) exhibited a significant difference between confirmed TB vs unconfirmed TB children but with a low sensitivity and specificity and CXCL10 (AUC 0.6011, sensitivity 63\%, specificity $51 \%$ ) exhibited no significant discriminatory power between confirmed TB vs unconfirmed TB children. Thus, plasma chemokines exhibit the potential to serve as biomarkers to distinguish both confirmed and unconfirmed TB disease from unlikely TB children.

\section{A plasma signature of two or three chemokines is an accurate biomarker discriminating confirmed or unconfirmed TB disease from unlikely TB}

A combinatorial analysis of multiple immune biomarkers was carried out to define the POC diagnostic accuracy of ideal marker combinations of the tested circulating plasma chemokines using the CombiROC method. This aims to examine the best combinations of biomarkers through a combined analysis of ROC curves, considering the sensitivity and specificity of all possible immune markers [17]. The examined chemokines as multiple marker signatures were evaluated in different combinations using CombiROC and the best combinations with 
the highest AUC, sensitivity and specificity were selected (Fig. 4). As shown in Fig. 4A, the dual combination of chemokines CCL1/CXCL1 (AUC 1, sensitivity 100\%, specificity 100\%) CCL1/CXCL10 (AUC 0.999, sensitivity 97\%, specificity 100\%), CXCL1/CXCL10 (AUC 0.999, sensitivity $100 \%$, specificity $97 \%$ ) and triple combination of chemokines CCL1/CXCL1/CXCL10 (AUC 1, sensitivity $100 \%$, specificity $100 \%$ ) showed significant discriminatory power with high AUC, sensitivity and specificity in discriminating confirmed TB from unlikely TB children. Similarly, as shown in Fig. 4B, the dual combination of chemokines CCL1/CXCL1 (AUC 0.966, sensitivity 87\%, specificity 96\%) CCL1/CXCL10 (AUC 0.990, sensitivity 97\%, specificity 92\%), CXCL1/CXCL10 (AUC 0.933, sensitivity $83 \%$, specificity $92 \%$ ) and triple combination of chemokines CCL1/CXCL1/CXCL10 (AUC 0.990, sensitivity $97 \%$, specificity $92 \%$ ) showed significant discriminatory power with high AUC, sensitivity and specificity in discriminating unconfirmed TB from unlikely TB children. Overall, we found that a biomarker signature of two or three chemokines exhibited excellent predictive performance in both confirmed and unconfirmed TB.

\section{Biomarkers discriminating active TB disease from unlikely TB}

Classification and regression trees (CART) models were employed to identify the cut-off for the biomarkers which separate the active TB disease from those with unlikely TB children. We have used the data from all the markers for the tree construction and then we selected the most relevant biomarker that classifies the group more accurately (Fig. 5). As shown in Fig. 5A, CCL1 with a cut-off value of $20.99 \mathrm{pg} / \mathrm{ml}$ was classified by the CART model, which demonstrated that the model was able to discriminate samples with the AUC 0.944, sensitivity 93\%, specificity $95 \%$ in confirmed $\mathrm{TB}$ vs unlikely $\mathrm{TB}$. As shown in Fig. 5B, CCL1 with cut-off value of $20.99 \mathrm{pg} / \mathrm{ml}$, CXCL10 cut-off value of $28.48 \mathrm{pg} / \mathrm{ml}$ and CXCL2 cut-off value of $85.27 \mathrm{pg} / \mathrm{ml}$ were classified by the CART model which determined that the model was able to discriminate samples with the AUC 0.920 , sensitivity $92 \%$, specificity $95 \%$ in unconfirmed TB vs unlikely TB. This CART analysis was able to demonstrate that CCL1 with a cut off value of $20.99 \mathrm{pg} / \mathrm{ml}$ is a sensitive diagnostic immune biomarker for diagnosis of active TB in children.

\section{Plasma chemokines levels are significantly diminished following ATT}

To elucidate whether the increased levels of chemokines are directly related with TB disease, we measured the levels of these chemokines in a subgroup of children with confirmed TB $(n=24)$ and unconfirmed TB $(n=23)$ before and after a standard course of ATT (Pre-Treatment (pre-T) versus Post-Treatment (post- $\mathrm{T}$ ). As shown in Fig. 6A, in children with confirmed TB at the end of ATT, the plasma levels of CCL1 (GM of $54.1 \mathrm{pg} / \mathrm{ml}$ in pre-T vs. $31.1 \mathrm{pg} / \mathrm{ml}$ in post-T), CXCL1 (GM of $147.8 \mathrm{pg} /$ $\mathrm{ml}$ in pre-T vs. $88.7 \mathrm{pg} / \mathrm{ml}$ in post-T), CXCL2 (GM of

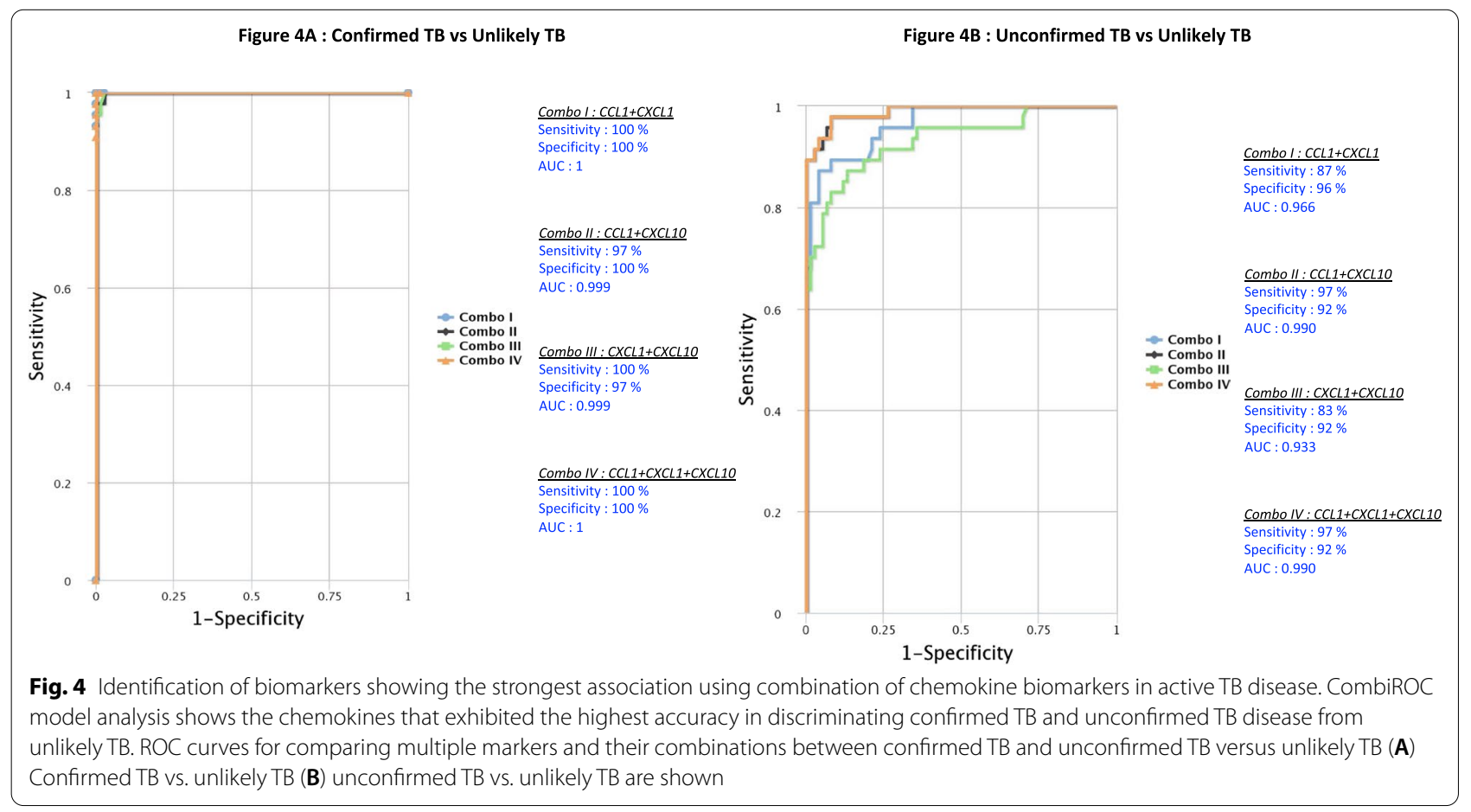


Figure 5A : Confirmed TB vs Unlikely TB
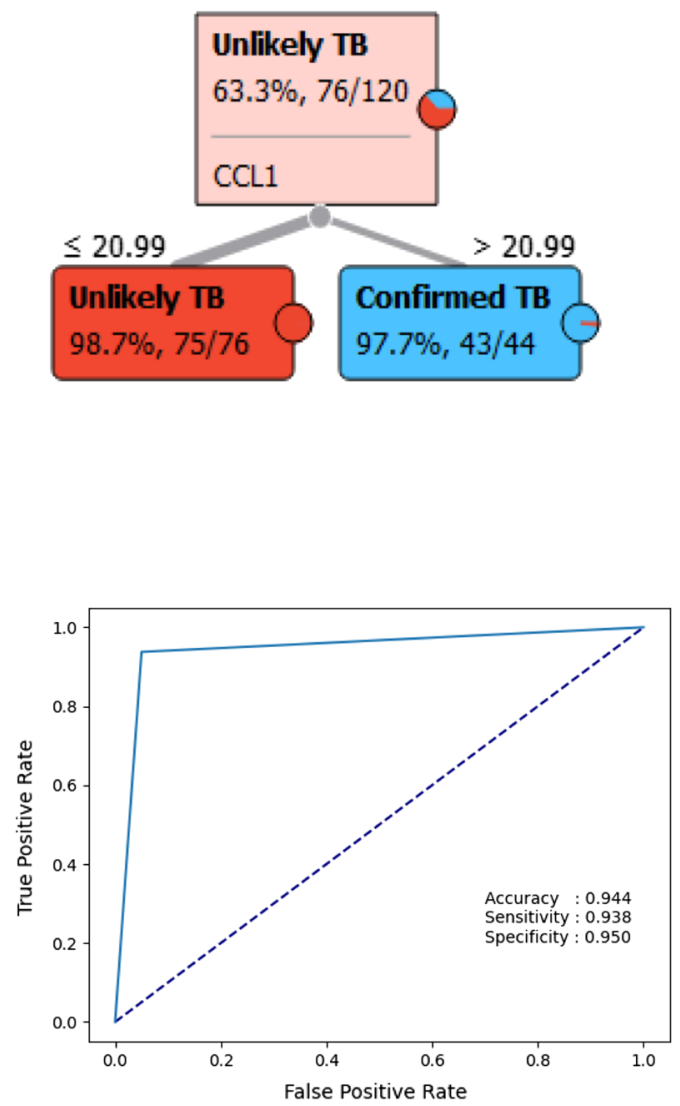

Figure 5B : Unconfirmed TB vs Unlikely TB

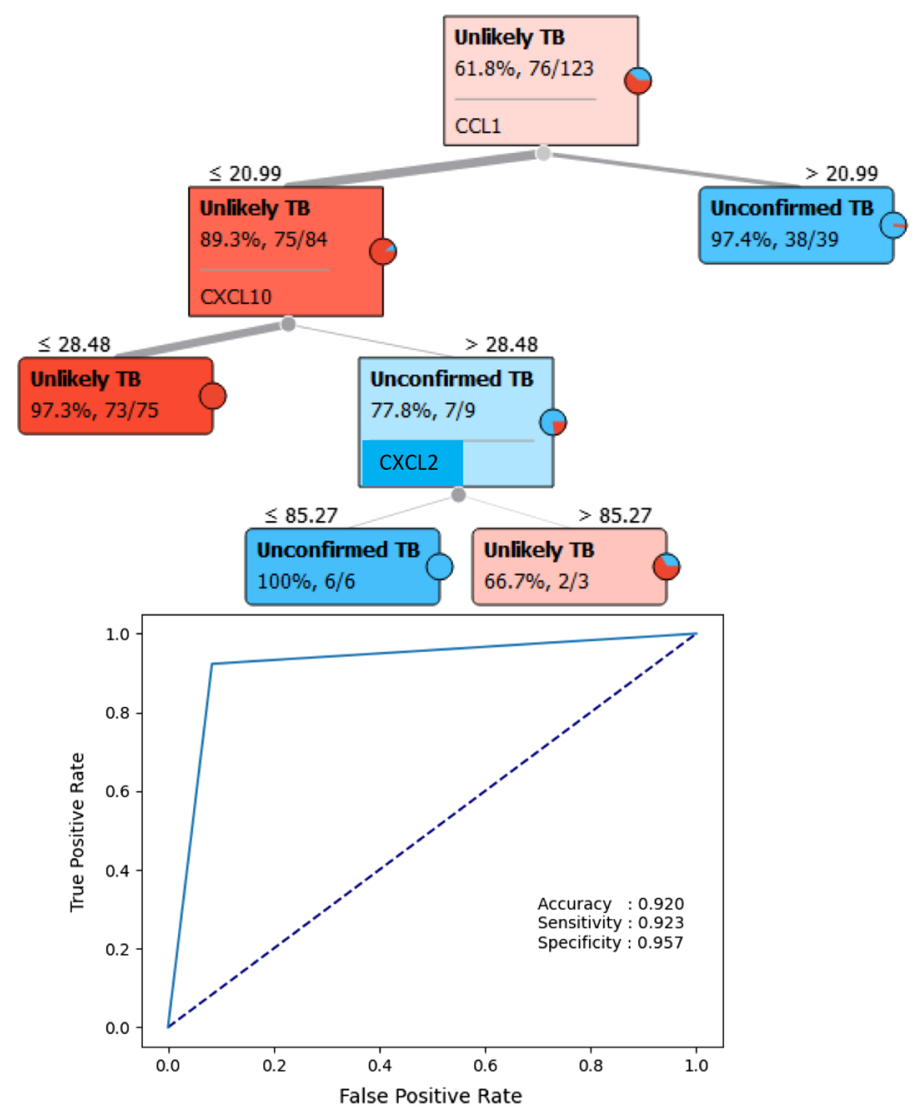

Fig. 5 Identification of biomarkers showing the strongest associations with active TB disease. CART model analysis shows the chemokines that exhibited the highest accuracy in discriminating (A) Confirmed TB vs unlikely and ROC curves were employed to quantify the accuracy of single or combined biomarkers. B Unconfirmed TB vs unlikely TB and ROC curves were employed to quantify the accuracy of single or combined biomarkers. Cut-off value of CCL1 is determined by this model

$768.2 \mathrm{pg} / \mathrm{ml}$ in pre-T vs. $370.1 \mathrm{pg} / \mathrm{ml}$ in post-T), CXCL10 (GM of $75.9 \mathrm{pg} / \mathrm{ml}$ in pre-T vs. $40.5 \mathrm{pg} / \mathrm{ml}$ in post-T) were significantly decreased in comparison to pre-treatment levels. Likewise, as shown in Fig. 6B, in children with unconfirmed TB at the end of ATT, the plasma levels of CCL1 (GM of $42.5 \mathrm{pg} / \mathrm{ml}$ in pre-T vs. $27.7 \mathrm{pg} / \mathrm{ml}$ in post-T), CCL11 (GM of $13 \mathrm{pg} / \mathrm{ml}$ in pre-T vs. $14.1 \mathrm{pg} /$ $\mathrm{ml}$ in post-T), CXCL1 (GM of $77.7 \mathrm{pg} / \mathrm{ml}$ in pre-T vs. $30.4 \mathrm{pg} / \mathrm{ml}$ in post-T) and CXCL10 (GM of $105 \mathrm{pg} / \mathrm{ml}$ in pre-T vs. $21.9 \mathrm{pg} / \mathrm{ml}$ in post-T) were also significantly diminished in comparison to pre-treatment levels. Next, we also wanted to assess whether the chemokines levels at the end of ATT in the confirmed TB group were similar to that of the unlikely TB group at baseline. As shown in Additional file 1: Fig. S1, children with confirmed TB at the end of ATT, exhibited levels of CCL1, CCL3, CCL4, CXCL1, CXCL2 and CXCL10, that were significantly increased while the levels of CCL11 and CXCL9 were significantly decreased in comparison to unlikely TB group.

\section{Discussion}

Pediatric populations are at a higher risk to progress to active TB disease following primary infection compared to adults [18]. Furthermore, extrapulmonary TB or disseminated forms of the TB disease are more common in young children [19]. Novel diagnostic biomarkers are still needed for the diagnosis of pediatric TB population and existing immune biomarker research for this target thus far has yielded partial success $[1,20,21]$. Despite the desperate need for better diagnosis of TB in children, only limited knowledge is currently available for understanding the mechanisms responsible for the disease severity during pediatric tuberculosis [22, 23]. Previously published studies on chemokines have prominently contributed to the understanding of TB pathogenesis and also 


\section{Figure 6A : Confirmed TB}

CCL1

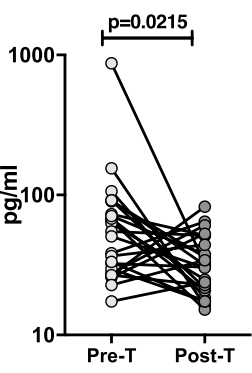

CXCL1

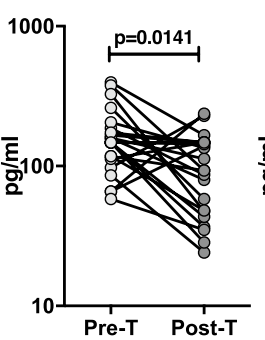

CCL2

CCL3

CCL4

CCL11
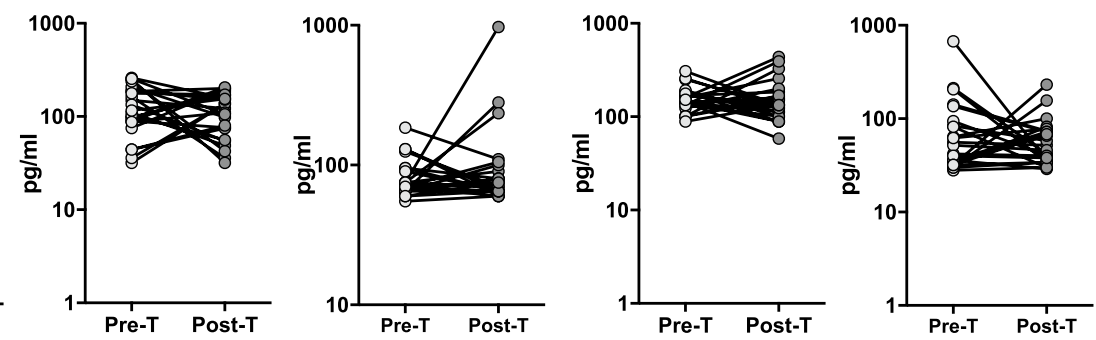

CXCL2

CXCL9
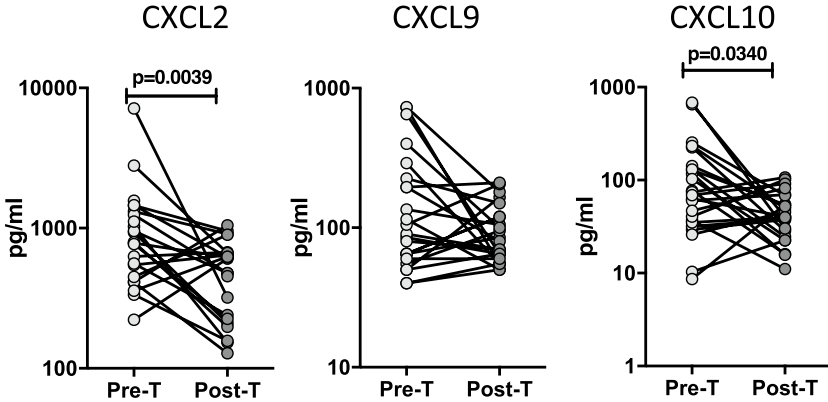

CXCL11

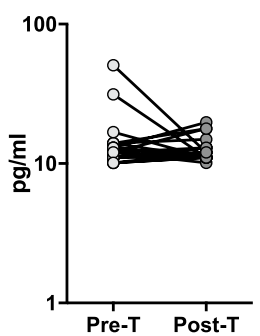

Figure 6B : Unconfirmed TB

CCL1
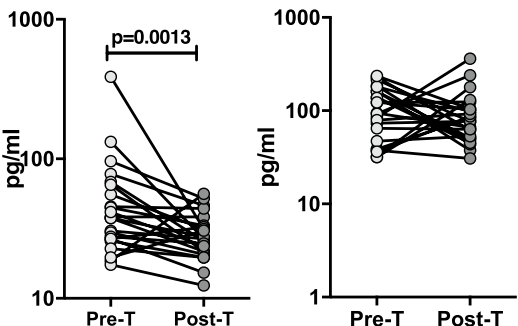

CXCL1
CCL2

Pre-T Post-T
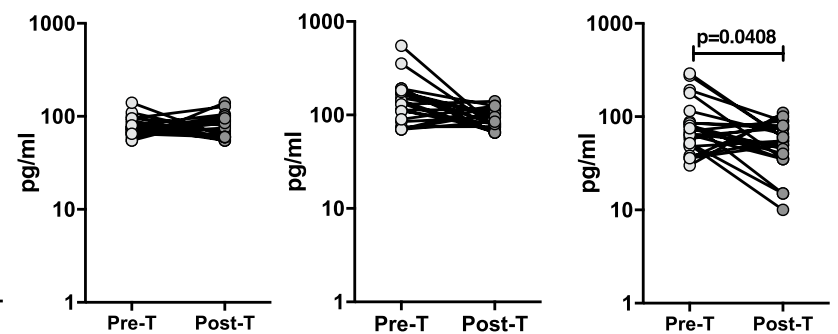

CXCL9

CXCL10

CXCL11
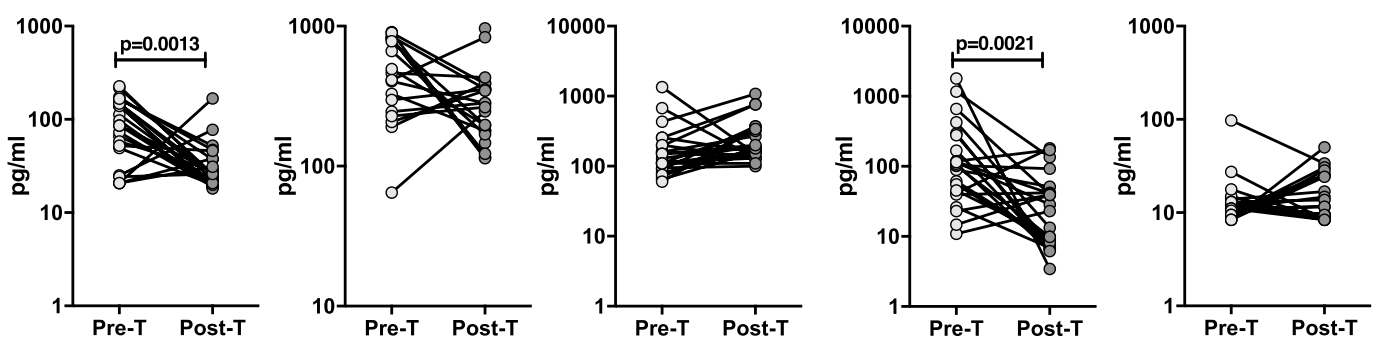

Fig. 6 Diminished plasma levels of chemokines at the end of standard anti-tuberculosis (TB) therapy in children with active TB disease. The plasma levels of CC and CXC chemokines at baseline (pre-T) and at 6 months of anti-TB treatment (post-T) in a subset of $(\mathbf{A})$ Confirmed TB ( $n=24)$ and $(\mathbf{B})$ Unconfirmed TB $(n=23)$. The data are presented as line graphs with each line representing a single individual 
findings from adult $\mathrm{TB}$ reported that chemokines might differentiate active TB from latent $\mathrm{TB}$ and healthy controls [11]. However, most of the studies in this field were performed in people more than 15 years old. Hence, in this study we sought to fill this knowledge gap by examining plasma chemokines in children with confirmed $\mathrm{TB}$, unconfirmed TB and unlikely TB. Our results add to the prevailing knowledge in the field by validating that plasma immune biomarkers like CCL1, CCL3, CXCL1, CXCL2 and CXCL10 can strikingly distinguish confirmed TB or unconfirmed TB from unlikely TB controls children in a highly endemic region. These blood-based immune biomarkers could perhaps be used as targets for a POC test without dependency on a complex laboratory arrangement.

Overall, results from our study exhibited that CC chemokines-CCL1 and CCL3 as well as the CXC chemokines-CXCL1, CXCL2 and CXCL10 are present at significantly higher concentrations in the plasma of active TB children in comparison to unlikely TB children but with other respiratory ailments. Interestingly no difference in CXCL9 was observed, which is different from what was reported previously in adults [12] and this could be attributable to differences in chemokine levels of adults and children. In addition, the plasma concentration of CXCL9 and CXCL10 were lower compare to other published studies due to the fact most of these studies are in adults [24].

For the recruitment of cells into the $M$.tb-infected lung, chemokines play a dominant role, which in turn leads to control of M.tb infection [25]. However, under certain conditions of lung damage and inflammation, chemokines drive the pathogenesis leading to severe TB disease $[25,26]$. Previous studies, including our own in adult $\mathrm{TB}$ patients, have reported results regarding the diagnostic potential of chemokine biomarkers to identify TB disease from those who are uninfected in a population with endemic TB setting [12]. Previous studies suggest that CXCL10 (IP-10) is a promising immune biomarker for diagnosis of either latent TB or active TB disease [27]. To support our existing data, a recent study in children with pediatric TB reported that CXCL10 (IP-10) showed a significant difference between the TB children (confirmed $\mathrm{TB}$ and unconfirmed $\mathrm{TB}$ ) when compared with unlikely TB children [28]. In addition, the authors of the study also reported that the combination of IP-10 with IL-7 and IL-1ra markers showed an average sensitivity of $70 \%$ and specificity of $75 \%$ [29], whereas in our study, the combination of CXCL10 with other chemokines such as CCL1 and CXCL1 showed 100\% sensitivity and specificity in confirmed TB and sensitivity of $97 \%$ and specificity of $92 \%$ in unconfirmed TB in comparison to unlikely
TB. Further, in order to test the diagnostic accuracy of these markers, ROC curve analysis was applied in the study population. The results show that CCL1, CXCL1 and CXCL10 could clearly discriminate confirmed TB children from unlikely TB controls with more than $90 \%$ sensitivity, specificity and good AUC, whereas CCL1 and CXCL10 alone showed a good sensitivity, specificity and AUC between unconfirmed TB and unlikely TB children. The results of this study further confirm that our diagnostic chemokine biomarkers perform well in this population with a greater background prevalence of LTB at the population level.

We have developed a three-immune biosignature of chemokines which could efficiently discriminate confirmed and unconfirmed TB from unlikely $\mathrm{TB}$ with good accuracy. Our current results report that three chemokine immune biomarkers (CCL1, CXCL10 and CXCL1) had the ability to discriminate between active TB disease and non-TB disease in children with high levels of sensitivity and specificity. Among these three chemokines, results from ROC and CART analysis reveals that CCL1 with a cut-off values of $21 \mathrm{pg} / \mathrm{ml}$ exhibits high levels of accuracy for the detection of active TB disease. Our findings clearly report that CCL1 is a stand-alone biomarker with ability to discriminate confirmed or unconfirmed TB from unlikely TB. In addition, CCL1 in combination of CXCL10 or CXCL1 will offer added advantage by increasing the sensitivity and specificity of the biomarker assays for further diagnosis. CCL1 will be further validated in a different endemic cohort for the development of a POC test, that can potentially be used as a future immunodiagnostic assay for pediatric TB. Our study also demonstrates that these chemokine biomarkers decrease in plasma levels following anti-TB treatment, confirming an association of these biomarkers with TB disease in children. In addition, our findings also revealed that at end of ATT in the confirmed TB group, most of the chemokine levels are still increased compared to the unlikely $\mathrm{TB}$ group indicating that the chemokines may take longer than six months to attain the normal range.

The strengths of this study involve the use of well characterised participant groups and, critically, the inclusion of an unlikely $\mathrm{TB}$ group, enabling us to determine the high sensitivity and specificity of the chemokine responses between the confirmed TB/ unconfirmed TB vs unlikely TB. Some of the previous studies have examined the chemokine response in antigen-stimulated samples from the pediatric TB population. However, the drawback of using antigenstimulated samples is the inconvenience in translating the assays to a point-of-care mode. Limitations of our 
study include a limited sample size, the absence of a validation cohort and the lack of inclusion of healthy control children. However, our results are promising enough to prompt further investigation of these biomarkers in large scale studies using diverse endemic populations. Hence, our study fills in the knowledge gap by performing profiling of plasma chemokines in unstimulated and stored plasma samples. Our study thus provides novel evidence for the utility of chemokines as biomarkers for pediatric TB.

\section{Abbreviations}

ATT: Anti-tuberculosis therapy; CART: Classification and regression trees; GM: Geometric mean; M.tb: Mycobacterium tuberculosis; POC: Point-of-care; ROC: Receiver operator characteristics; TST:Tuberculin skin; TB: Tuberculosis; WHO: World Health Organization.

\section{Supplementary Information}

The online version contains supplementary material available at https://doi. org/10.1186/s12879-021-06749-6.

Additional file 1: Figure $\mathbf{S 1}$. The plasma levels of CCL1, CCL2, CCL3, CCL4, CCL11, CXCL1, CXCL2, CXCL9, CXCL10 and CXCL11 were measured in confirmed TB individuals at 6 months of anti-TB treatment (post-T) $(n=24)$ and unlikely TB $(n=76)$ individuals at baseline. The data are represented as scatter plots with each circle representing a single individual. $P$ values were calculated using the Mann-Whitney test.

\section{Acknowledgements}

We thank the staffs of Department of Clinical Research, NIRT - Dr. Annie Studhi, Dr. Bency Joseph, Gunasundari A, Valarmathi Nagarajan, Mangalambal, Shakila Shankar, Stella Mary A, Selvi R, Jemima J, Krishna Yadav, Vaishnavi S, Hema Giranab, Sita A, Bharathi S, Vedavalli M, Shanthi M, Dino Xavier, Guru K, Manjith Kumar, Sananthya, and Sasi Kumar for valuable assistance in recruitment and followup of participants. We also thank Institute of Child Health and Hospital for Children, Chennai; Government Stanley Medical College and Hospital, Chennai, for valuable assistance in recruiting the participants for this study.

\section{Authors' contributions}

Designed the study (S.B., N.P.K., S.H); conducted experiments (N.P.K., G.N.S.); acquired data (N.P.K., K.T.); analyzed data (N.P.K., K.T.); contributed reagents and also revised subsequent drafts of the manuscript (S.H., S.S., S.T., S.B.); responsible for the enrolment of participant and also contributed to acquisition and interpretation of clinical data (V.V.B., S.B., E.S., G.J., A.M.A., D.B.); wrote the manuscript (S.B., N.P.K.). All authors read and approved the final manuscript.

\section{Funding}

This work was supported by the Division of Intramural Research, National Institute of Allergy and Infectious Diseases (NIAID). And this study was also partially supported by USAID, WHO and ICMR under Model DOTS project. The funders had no role in study design, data collection and analysis, decision to publish, or preparation of the manuscript.

\section{Availability of data and materials}

The datasets used and/or analysed during the current study are available from the corresponding author on reasonable request.

\section{Declarations}

Ethics approval and consent to participate

All individuals were examined as part of a clinical research protocol (NIRTIEC2012004) approved by Institutional Ethics committees of the
ICMR-National Institute for Research in Tuberculosis, Chennai and all methods were performed in accordance with the relevant guidelines and regulations. All legal guardians of the included children provided written informed consent.

\section{Consent for publication}

Not applicable in this section.

\section{Competing interests}

The authors declare that they have no competing interests.

\section{Author details}

${ }^{1}$ International Center for Excellence in Research, National Institute for Research in Tuberculosis, Chennai, India. ${ }^{2}$ ICMR-National Institute for Research in Tuberculosis, Chennai, India. Institute of Child Health and Hospital for Children, Chennai, India. ${ }^{4}$ Government Stanley Medical College and Hospital, Chennai, India. ${ }^{5}$ World Health Organisation, Geneva, Switzerland. ${ }^{6}$ LPD, NIAID, NIH, Bethesda, MD, USA.

Received: 16 April 2021 Accepted: 10 September 2021

Published online: 11 October 2021

\section{References}

1. Portevin D, Moukambi F, Clowes P, Bauer A, Chachage M, Ntinginya NE, Mfinanga E, Said K, Haraka F, Rachow A, et al. Assessment of the novel T-cell activation marker-tuberculosis assay for diagnosis of active tuberculosis in children: a prospective proof-of-concept study. Lancet Infect Dis. 2014;14(10):931-8.

2. Perez-Velez CM, Marais BJ. Tuberculosis in children. N Engl J Med. 2012;367(4):348-61

3. Marais BJ, Graham SM, Maeurer M, Zumla A. Progress and challenges in childhood tuberculosis. Lancet Infect Dis. 2013;13(4):287-9.

4. WHO: High-priority target product profiles for new tuberculosis diagnostics. In.; 2014.

5. Basu Roy R, Whittaker E, Seddon JA, Kampmann B. Tuberculosis susceptibility and protection in children. Lancet Infect Dis. 2019;19(3):e96-108.

6. Glaziou P, Floyd K, Raviglione MC. Global epidemiology of tuberculosis. Semin Respir Crit Care Med. 2018;39(3):271-85.

7. Seddon JA, Whittaker E, Kampmann B, Lewinsohn DA, Osman M, Hesseling AC, Rustomjee R, Amanullah F. The evolving research agenda for paediatric tuberculosis infection. Lancet Infect Dis. 2019;19(9):e322-9.

8. Latorre I, Diaz J, Mialdea I, Serra-Vidal M, Altet N, Prat C, Diez N, Escribano A, Casas I, Rodrigo C, et al. IP-10 is an accurate biomarker for the diagnosis of tuberculosis in children. J Infect. 2014:69(6):590-9.

9. Ruhwald M, Bodmer T, Maier C, Jepsen M, Haaland MB, Eugen-Olsen J, Ravn P. Tbnet: evaluating the potential of IP-10 and MCP-2 as biomarkers for the diagnosis of tuberculosis. Eur Respir J. 2008;32(6):1607-15.

10. Chegou NN, Sutherland JS, Namuganga AR, Corstjens PL, Geluk A, Gebremichael G, Mendy J, Malherbe S, Stanley K, van der Spuy GD, et al. Africa-wide evaluation of host biomarkers in QuantiFERON supernatants for the diagnosis of pulmonary tuberculosis. Sci Rep. 2018;8(1):2675.

11. Mihret A, Bekele Y, Bobosha K, Kidd M, Aseffa A, Howe R, Walzl G. Plasma cytokines and chemokines differentiate between active disease and nonactive tuberculosis infection. J Infect. 2013;66(4):357-65.

12. Kumar NP, Moideen K, Nancy A, Viswanathan V, Shruthi BS, Sivakumar S, Natarajan M, Kornfeld H, Babu S. Plasma chemokines are biomarkers of disease severity, higher bacterial burden and delayed sputum culture conversion in pulmonary tuberculosis. Sci Rep. 2019;9(1):18217.

13. Graham SM, Cuevas LE, Jean-Philippe P, Browning R, Casenghi M, Detjen AK, Gnanashanmugam D, Hesseling AC, Kampmann B, Mandalakas A, et al. Clinical case definitions for classification of intrathoracic tuberculosis in children: an update. Clin Infect Dis. 2015;61(Suppl 3):S179-187.

14. Kumar NP, et al. Discovery and validation of a three-cytokine plasma signature as a biomarker for diagnosis of pediatric tuberculosis. Front Immunol. 2021. https://doi.org/10.3389/fimmu.2021.653898.

15. Motulsky HJ, Brown RE. Detecting outliers when fitting data with nonlinear regression - a new method based on robust nonlinear regression and the false discovery rate. BMC Bioinform. 2006;7:123. 
16. Mazzara S, Rossi RL, Grifantini R, Donizetti S, Abrignani S, Bombaci M. CombiROC: an interactive web tool for selecting accurate marker combinations of omics data. Sci Rep. 2017;7:45477.

17. Rathnayake D, Chang T, Udagama P. Selected serum cytokines and nitric oxide as potential multi-marker biosignature panels for Parkinson disease of varying durations: a case-control study. BMC Neurol. 2019;19(1):56.

18. Thomas TA. Tuberculosis in children. Thorac Surg Clin. 2019;29(1):109-21.

19. Yang Z, Kong Y, Wilson F, Foxman B, Fowler AH, Marrs CF, Cave MD, Bates $\mathrm{JH}$. Identification of risk factors for extrapulmonary tuberculosis. Clin Infect Dis. 2004;38(2):199-205.

20. Armand M, Chhor V, de Lauzanne A, Guerin-El Khourouj V, Pedron B, Jeljeli M, Gressens P, Faye A, Sterkers G. Cytokine responses to quantiferon peptides in pediatric tuberculosis: a pilot study. J Infect. 2014;68(1):62-70.

21. Chiappini E, Della Bella C, Bonsignori F, Sollai S, Amedei A, Galli L, Niccolai E, Del Prete G, Singh M, D'Elios MM, et al. Potential role of M. tuberculosis specific IFN-gamma and IL-2 ELISPOT assays in discriminating children with active or latent tuberculosis. PLoS ONE. 2012;7(9): e46041.

22. Lighter-Fisher J, Peng $\mathrm{CH}$, Tse DB. Cytokine responses to QuantiFERON(R) peptides, purified protein derivative and recombinant ESAT-6 in children with tuberculosis. Int J Tuberc Lung Dis. 2010;14(12):1548-55.

23. Kumar NP, Anuradha R, Suresh R, Ganesh R, Shankar J, Kumaraswami V, Nutman TB, Babu S. Suppressed type 1, type 2, and type 17 cytokine responses in active tuberculosis in children. Clin Vaccine Immunol. 2011;18(11):1856-64.
24. Manyelo CM, Solomons RS, Snyders Cl, Mutavhatsindi H, Manngo PM, Stanley K, Walzl G, Chegou NN. Potential of host serum protein biomarkers in the diagnosis of tuberculous meningitis in children. Front Pediatr. 2019;7:376.

25. Monin L, Khader SA. Chemokines in tuberculosis: the good, the bad and the ugly. Semin Immunol. 2014;26(6):552-8.

26. Slight SR, Khader SA. Chemokines shape the immune responses to tuberculosis. Cytokine Growth Factor Rev. 2013;24(2):105-13.

27. Sudbury EL, Clifford V, Messina NL, Song R, Curtis N. Mycobacterium tuberculosis-specific cytokine biomarkers to differentiate active TB and LTBI: a systematic review. J Infect. 2020;81(6):873-81.

28. Qiu X, Tang Y, Yue Y, Zeng Y, Li W, Qu Y, Mu D. Accuracy of interferongamma-induced protein 10 for diagnosing latent tuberculosis infection: a systematic review and meta-analysis. Clin Microbiol Infect. 2019;25(6):667-72.

29. Togun T, Hoggart CJ, Agbla SC, Gomez MP, Egere U, Sillah AK, Saidy B, Mendy F, Pai M, Kampmann B. A three-marker protein biosignature distinguishes tuberculosis from other respiratory diseases in Gambian children. EBioMedicine. 2020;58: 102909.

\section{Publisher's Note}

Springer Nature remains neutral with regard to jurisdictional claims in published maps and institutional affiliations.
Ready to submit your research? Choose BMC and benefit from:

- fast, convenient online submission

- thorough peer review by experienced researchers in your field

- rapid publication on acceptance

- support for research data, including large and complex data types

- gold Open Access which fosters wider collaboration and increased citations

- maximum visibility for your research: over 100M website views per year

At BMC, research is always in progress.

Learn more biomedcentral.com/submissions 